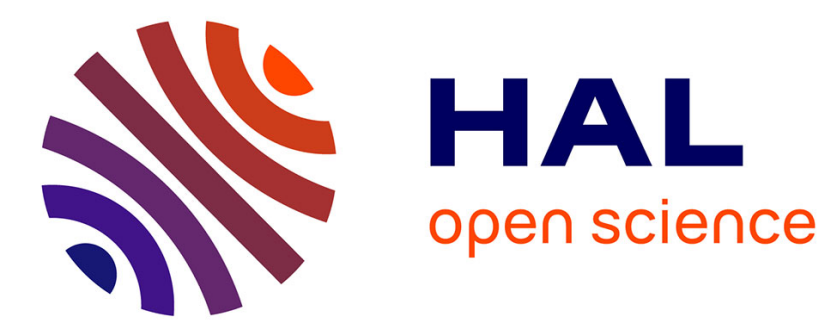

\title{
Continuously-implemented sliding-mode adaptive unknown-input observers under noisy measurements
}

\author{
Habib Dimassi, Antonio Loria, Safya Belghith
}

\section{To cite this version:}

Habib Dimassi, Antonio Loria, Safya Belghith. Continuously-implemented sliding-mode adaptive unknown-input observers under noisy measurements. Systems and Control Letters, 2012, 61 (12), pp.1194-1202. 10.1016/j.sysconle.2012.09.002 . hal-00831380

\section{HAL Id: hal-00831380 \\ https://hal.science/hal-00831380}

Submitted on 7 Jun 2013

HAL is a multi-disciplinary open access archive for the deposit and dissemination of scientific research documents, whether they are published or not. The documents may come from teaching and research institutions in France or abroad, or from public or private research centers.
L'archive ouverte pluridisciplinaire HAL, est destinée au dépôt et à la diffusion de documents scientifiques de niveau recherche, publiés ou non, émanant des établissements d'enseignement et de recherche français ou étrangers, des laboratoires publics ou privés. 


\title{
Continuously-implemented Sliding-mode Adaptive Unknown-input Observers under Noisy measurements
}

\author{
Habib Dimassi ${ }^{a, c} \quad$ Antonio Loría $^{a}$ Safya Belghith ${ }^{\mathrm{b}}$ \\ ${ }^{\mathrm{a}} \mathrm{LSS}$-SUPELEC, CNRS, 3, Rue Joliot Curie, 91192 Gif s/Yvette, France \\ ' Sys'Com-Ecole Nationale d'Ingénieurs de Tunis, Tunisia \\ ${ }^{\mathrm{c}}$ Univ Paris Sud, Orsay, France and Sys'Com-Ecole Nationale d'Ingénieurs de Tunis, Tunisia
}

\begin{abstract}
We propose an estimator for nonlinear systems with unmatched unknown inputs and under measurement noise. The estimator design is based on the combination of observer design for descriptor systems, sliding-modes theory and adaptive control. The estimation of the measurement noise is achieved thanks to the transformation of the original system into a singular form where the measurement noise makes part of the augmented state. Two adaptive parameters are updated online, one to compensate for the unknown bounds on the states, the unkown inputs and the measurement noise and a second one to compensate for the effect of the nonlinearities. To join robust state estimation and unknown-inputs reconstruction, our approach borrows inpiration from sliding-mode theory however, all signals are comtinuously implemented. We demonstrate that both state and unknown-inputs estimation are achieved up to arbitrarily small tolerance. The utility of our theoretical results is illustrated through simulation case-studies.
\end{abstract}

Keywords: Sliding modes, unknown-input observer, stability.

\section{Introduction}

One of the most challenging obstacles in observer design is to deal with systems that are nonlinear in the unmeasured variables. A large number of articles have been written on the subject based on various types of conditions such as: (global) Lipschitz conditions -[1], sector conditions -[3], boundedness of nonlinearities -[4], boundedness of state trajectories -[19], high-gain designs -[2], sliding-mode design -[30].

Sliding-mode observer design consists in defining an attractive sliding manifold and using discontinuous inputs to steer the estimation error trajectories to this manifold in finite time. Without doubt one attractive feature of slidingmode design is its ability to reject disturbances exactly, albeit its sensitivity to measurement noise. Regarding linear systems, see for instance [27] for a Lyapunov-based result and [12] for a method based on the ability to transform the system into a canonical form. It is commonly assumed that the unknown input is upper-bounded; in the case that the upper-bound is unknown, parameter adaptation is typically used-see e.g., [28, 13, 29].

Relying on literature on the left-invertibility problem -[18] sliding-mode design for linear systems has also been extended to the realm of nonlinear systems with unknown inputs, via homogeneous transformations and output injection, to obtain an observability normal form -see for instance [5]. One of the advantages of this method is that it applies to systems with high relative degree (between the unknown input and the measured output) then, the system is transformed to a canonical form where the unknown inputs appear only in the 'last' equation (this assumption is

Email addresses: dimassi@lss.supelec.fr (Habib Dimassi), loria@lss.supelec.fr (Antonio Loría), safya.belghith@enit.rnu.tn (Safya Belghith). 
sometimes called observability matching condition). The method has been successfully used in the design of high-order sliding mode estimators; in this case, one may establish finite-time estimation and unknown input reconstruction recursively (step by step). See for instance [6, 14] as well as the works on high-order differentiators by [20, 21]. The main and clear disadvantage of this method is that it relies on coordinate transformations which are valid locally.

In this paper, we propose a continuously-implemented sliding-mode estimator for nonlinear systems with "Lipschitz" nonlinearities ${ }^{1}$ such that the Lipschitz constant is unknown, unknown inputs and measurement noise. Both states, unknown inputs and measurement noise are assumed bounded with unknown upper-bounds. To the best of our knowledge, the problem of joint robust state estimation and unknown-inputs reconstruction in such scenario has not been solved yet; in particular, the problem of designing sliding mode observers for systems with unknown inputs and measurement noise simultaneously remains open. In [12] the authors consider linear systems with unknown inputs and measurement noise but not simultaneously and although the recent $\operatorname{article}^{2}$ [15] is a rare exception since noise and unknown inputs are handled simultaneously, this work is limited to linear systems and it is also assumed that the same additive perturbation affects both the dynamics and the outputs.

Our estimation approach consists in three main parts, in accordance with the system's structure: first, we transform the original system into a descriptor form by considering the measurement noise as a supplementary state variable (this idea is reminiscent of the design of augmented-state observers to cope with the unknown inputs as additional states -see [23, 24]), then we design an observer for the 'nominal' linear part of considered descriptor system. Secondly, we use terms inspired from sliding-mode theory as well as the concept of the equivalent control to reconstruct the unknown inputs. Our method is reminiscent of conventional first-order sliding-mode unknown-input observers except that we replace the commonly-used discontinuous functions by smooth saturations; also, some of our technical conditions such as the relative-degree-one assumption, recall those in [11] and subsequent work by the authors. However, our estimator design approach does not rely on coordinate transformations. Thirdly, based on the Lyapunov theory, we incorporate an adaptation law to compensate for some constant depending on the unknown upper-bounds on the states, the unknown inputs and the measurement noise; similarly, adaptation is used to cope with the effect of the Lipschitz nonlinearities.

From a technical viewpoint, the use of adaptation comes at the price of non-zero arbitrarily small estimation errors. The establishment of our main result relies on a fundamental but intuitive lemma on high-gain design which follows from [16], we prove that the estimation errors converge arbitrarily close to the sliding manifold and then, to a neighborhood of the origin. Consequently, the states, the unknown inputs and the additive output noise may be reconstructed with arbitrary accuracy.

The remainder of the paper is organized as follows. In the following section we formulate the problem; in Section 2 we present a preliminary result in anticipation of our main theorem; the latter is presented in Section 3 . Then, two numeric examples illustrative of the utility of our theoretical findings are discussed in Section 4 and we wrap up the paper with conclusive remarks, in Section 5.

\subsection{Problem statement}

We solve the state and input estimation problem under measurement noise for nonlinear systems of the form

$$
\begin{aligned}
\dot{x}_{*} & =A_{0} x_{*}+B f_{0}\left(x_{*}\right)+F \eta_{1}(t) \\
y & =C_{0} x_{*}+G_{0} \eta_{2}(t) .
\end{aligned}
$$

The problem consists in designing a dynamical system to reconstruct the unmeasured state vector $x_{*} \in \mathbb{R}^{n}$ as well as the unknown input $\eta_{1} \in \mathbb{R}^{q_{1}}$. It is assumed that the measured output $y \in \mathbb{R}^{p}$ is contaminated by additive noise, denoted by $\eta_{2} \in \mathbb{R}^{q_{2}}$. The function $f_{0}: \mathbb{R}^{n} \rightarrow \mathbb{R}^{s}$ is assumed to be once continuously differentiable.

Systems of the form (1) include those studied for instance in [30, 12]. Under the assumption that either $\eta_{1}$ or $\eta_{2}$ equals zero the authors of the latter show that the system may be decomposed in two parts, one which is not affected by the unknown inputs and another which generates the measured output; thereby making it unnecessary to design

\footnotetext{
${ }^{1}$ As it will be clear later, we do not assume that the nonlinearities are (globally) Lipschitz but we use boundedness of trajectories to perform the so-called Lipschitz transformation. See p. 3.

2 This reference appeared after the submission of our article.
} 
an unknown-input observer. In contrast, in this paper it is assumed that the system is nonlinear and both $q_{1}>0$ and $q_{2}>0$.

In the construction of unknown-input estimators in the absence of measurement noise, it is often assumed (see e.g., $[30,26])$ that the dimension of the unknown inputs does not exceed that of the measured functions that is, $q_{1} \leq p$. This is necessary to satisfy the detectability condition $\operatorname{rank}\left(C_{0} F\right)=\operatorname{rank}(F)$. In turn, the latter is necessary in most unknown-input observers designs - see e.g. [25]; it is also used in Lyapunov-based designs as in e.g., [27] and [16] or in order to decompose the system, as in [30] and [12]. A notable exception (for linear systems) is [14] where the authors propose a method to transform the system into a new canonical form; however, even though the authors of [14] succeed in avoiding the relative degree one assumption, it is assumed that measurements are noise-free i.e., $y=C_{0} x_{*}$. This work was extended in [6] to the case of nonlinear systems, yet locally transformed into a specific triangular observable form, for which a higher order sliding mode observer was introduced.

In the present setting that is, with measurement noise, we also need to impose that $q_{2} \leq p$ which is a necessary condition for $G_{0}$ to be of full rank. In turn, the latter is needed to guarantee the feasibility of the estimator.

Thus, we make the following standing hypothesis,

\section{Assumption 1.1}

a) $\eta_{1}, \eta_{2}$ and $y$ are such that $q_{1}+q_{2} \leq p$;

b) $F$ and $G_{0}$ are full-column-rank and $\operatorname{rank}\left(C_{0} F\right)=\operatorname{rank}(F)$;

c) the unknown inputs $\eta_{1}(t)$ and $\eta_{2}(t)$ are bounded and with bounded first derivatives;

d) the solutions $x_{*}(t)$ of (1) are forward complete and uniformly bounded.

The constraint $q_{1}+q_{2} \leq p$ in Condition 1.1a) is imposed as a sufficient condition for both $q_{1} \leq p$ and $q_{2} \leq p$ to hold simultaneously. In practice, this assumption is achievable if it is assumed that not all outputs are corrupted by the noise or all the measurements are affected by the same noise signal. Although this constraint clearly restricts the class of systems under consideration, it is necessary in a number of examples.

Condition 1.1c) is a harmless technical condition, recurrent in sliding-mode literature.

Condition 1.1d) is used to apply the so-called Lipschitz transformation on $f_{0}$. Let ${ }^{3} x_{*}=\left[x_{* 1}, \ldots, x_{* n}\right]^{\top}, \Omega=\left\{x_{*} \in\right.$ $\left.\mathbb{R}^{n},\left|x_{* i}\right| \leq \omega_{i}, 1 \leq i \leq n\right\}$ for a set of $n$ given numbers $\omega_{i}>0$. Let $\varsigma: \mathbb{R}^{n} \rightarrow \Omega$ be a locally linear saturation function that is, such that $\varsigma\left(x_{*}\right)=x_{*}$ for all $x_{*} \in \Omega$ and $\left|\varsigma\left(x_{* i}\right)\right|=1$ elsewhere, for each component of $x_{*}$. Then, define $f_{1}: \mathbb{R}^{n} \rightarrow \mathbb{R}^{s}$ such that $f_{1}\left(x_{*}\right)=f_{0}\left(\varsigma\left(x_{*}\right)\right)$; we obtain $f_{1}\left(x_{*}\right)=f_{0}\left(x_{*}\right)$ for each $x_{*} \in \Omega$. Hence, applying the mean value theorem, we can show that $f_{1}$ is globally Lipschitz with Lipschitz constant $K_{f}$ i.e.,

$$
\left|f_{1}\left(x_{1}\right)-f_{1}\left(x_{2}\right)\right| \leq K_{f}\left|x_{1}-x_{2}\right| \quad \forall x_{1}, x_{2} \in \mathbb{R}^{n}
$$

It follows that for all $t$ such that $x_{*}(t) \in \Omega$, the trajectories of the system (1) coincide with those of

$$
\begin{aligned}
\dot{x}_{*} & =A_{0} x_{*}+B f_{1}\left(x_{*}\right)+F \eta_{1}(t) \\
y & =C_{0} x_{*}+G_{0} \eta_{2}(t) .
\end{aligned}
$$

Hence, without loss of generality we assume that item (d) of Assumption 1.1 holds for the compact set $\Omega$ used above. The estimator design problem for (1) reduces to that for (3).

Next, let us introduce the extended state $x=\left[x_{*}^{\top}, \eta_{2}^{\top}\right]^{\top}$ and correspondingly,

$$
A=\left[\begin{array}{ll}
A_{0} & 0
\end{array}\right], \quad C=\left[\begin{array}{ll}
C_{0} & G_{0}
\end{array}\right], \quad T=\left[\begin{array}{ll}
I_{n} & 0
\end{array}\right]
$$

and $f: \mathbb{R}^{n+q_{2}} \rightarrow \mathbb{R}^{s}$ such that $f(x)=f_{1}\left(x_{*}\right)$. Then, the equations (3) take the descriptor-system form

$$
\begin{aligned}
T \dot{x} & =A x+B f(x)+F \eta_{1}(t) \\
y & =C x .
\end{aligned}
$$

\footnotetext{
$\overline{3}$ The symbol $|\cdot|$ stands for the (generalized) norm of elements in $\mathbb{R}^{n \times m}$ for any $n \geq 1$ and $m \geq 1$.
} 
Our motivation to address this problem lies on synchronization of chaotic systems used to encode information to be transmitted -see the example in Section 4. However, this problem is of broader interest, as for instance in the context of fault-detection -see [8,23] or estimation of switching rules in certain hybrid systems -see [22]. In the first two references, the state is augmented by the faults (unknown inputs); measurements are noise-free.

Our main result consists in an estimator for system (4). This is presented in Section 3; in anticipation of which, preliminary results are presented next.

\section{Sliding-mode unknown-input observer}

The estimator consists in three main parts. Firstly, we construct an observer for the "nominal" linear part $T \dot{x}=A x$. Secondly, adaptive high-gain is added to compensate for the Lipschitz nonlinearity $B f(x)$. Thirdly, we use slidingmode based correction terms to estimate the unknown inputs $\eta_{1}$.

\subsection{An observer for $T \dot{x}=A x$}

Let $P: \mathbb{R}^{n+q_{2} \times n}$ and $E: \mathbb{R}^{n+q_{2} \times p}$ be two constant design matrices such that

$$
P T=I+E C
$$

for given matrices $C$ and $T$. To investigate the solubility of (5) we re-write it in the form

$$
\left[\begin{array}{ll}
P & E
\end{array}\right]\left[\begin{array}{c}
T \\
-C
\end{array}\right]=I_{n+q_{2}}
$$

where $I_{n+q_{2}}$ denotes the identity matrix of dimension $n+q_{2}$. Equation $(6)$ is of the form $X R_{1}=R_{2}$ with $R_{1}=\left[\begin{array}{c}T \\ -C\end{array}\right]$, $R_{2}=I_{n+q_{2}}$ and $X=[P E]$. The latter Equation is solvable for $X$ if and only if

$$
\operatorname{Rank}\left[\begin{array}{c}
R_{1} \\
R_{2}
\end{array}\right]=\operatorname{Rank}\left[R_{1}\right]
$$

Moreover, for any arbitrary matrix $Z_{a}$ the solution is

$$
X=R_{2} R_{1}^{+}-Z_{a}\left(I-R_{1} R_{1}^{+}\right)
$$

where $R_{1}^{+}$denotes the generalized inverse of $R_{1}$. In turn, applied to the solution of (6) the condition (7) is

$$
\operatorname{Rank}\left[\begin{array}{cc}
I_{n} & 0 \\
-C_{0} & -G_{0} \\
I_{n} & 0 \\
0 & I_{q_{2}}
\end{array}\right]=\operatorname{Rank}\left[\begin{array}{cc}
I_{n} & 0 \\
-C_{0} & -G_{0}
\end{array}\right]
$$

which holds if and only if $G_{0}$ is full rank - cf. Assumption 1.1b) and [10, 7].

Now we pose the following natural choice of an estimator for the "nominal" descriptor system $T \dot{x}=A x$,

$$
P T \dot{\hat{x}}=P A \hat{x}-v_{1}
$$

where $v_{1}$ represents correcting additional terms yet to be determined so that the origin is an exponentially stable equilibrium for the estimation error equation

$$
P T(\dot{x}-\dot{\hat{x}})=P A(x-\hat{x})+v_{1} .
$$


What is more, define $e=x-\hat{x}$ then, we look for $v_{1}$ such that (10) become

$$
\dot{e}=K e
$$

where $K$ is Hurwitz. Using (5) in (10) we obtain

$$
\begin{aligned}
\dot{e}+E C \dot{e} & =P A e+v_{1} \\
\dot{e}+E \dot{y}-E C \dot{\hat{x}} & =P A e+v_{1}-\dot{\hat{x}}+\dot{\hat{x}} \\
\dot{e} & =P A e+v_{1}-E \dot{y}+(E C+I) \dot{\hat{x}}-\dot{\hat{x}} \\
& =P A e+v_{1}+P T \dot{\hat{x}}-(E \dot{y}+\dot{\hat{x}}) .
\end{aligned}
$$

Define $z:=E y+\hat{x}$ then

$$
\dot{e}=P A e+v_{1}+P T \dot{\hat{x}}-\dot{z}
$$

Now, let $L$ be such that

$$
K=P A-L C
$$

is Hurwitz. Such matrix L, i.e. satisfying (12), exists provided that the pair $(A, C)$ is observable; its numerical computation on a case-by-case basis, constitutes a standard pole-placement problem. So we see that by imposing

$$
v_{1}=-L C e+\dot{z}-P T \dot{\hat{x}}
$$

we obtain (11), which is exponentially stable at the origin.

Now we show how to implement (13) which in view of (9), is equivalent to $P A \hat{x}=-L C e+\dot{z}$. The latter holds if and only if

$$
\begin{aligned}
\dot{z} & =P A z-P A E y+L C e \\
& =P A z-P A E y-L C \hat{x}+L C x \\
& =P A z-P A E y-L C(z-E y)+L C x \\
& =K z-K E y+L y \\
& =K z+(L-K E) y .
\end{aligned}
$$

In summary, the estimator

$$
\begin{aligned}
& \dot{z}=K z+J y, \quad J=L-K E \\
& \hat{x}=z-E y
\end{aligned}
$$

with $L$ such that $P A-L C=K$ is Hurwitz, yields the estimation error dynamics (11) whose null solution is exponentially stable -see also [7].

\subsection{Adaptive compensation of nonlinearities}

Next, we consider the system (4) with $f$ globally Lipschitz (after a Lipschitz transformation) with constant $K_{f}$, considered to be unknown. We modify the estimator (9) designed for $T \dot{x}=A x$, by introducing the compensation terms $H_{1} f(\hat{x}), H_{2} u$ and $\frac{1}{2} \hat{\beta} H_{1} M(y-C \hat{x})$, with $H_{1}:=P B$ and $H_{2}=P F$. In place of (9), let

$$
P T \dot{\hat{x}}=P A \hat{x}-v_{1}+\frac{1}{2} \hat{\beta} H_{1} M(y-C \hat{x})+H_{1} f(\hat{x})+H_{2} u
$$

and equivalently, let the input (13) be implemented via the estimator

$$
\begin{aligned}
& \dot{z}=K z+J y+H_{1} f(\hat{x})+\frac{1}{2} \hat{\beta} H_{1} M(y-C \hat{x})+H_{2} u \\
& \hat{x}=z-E y
\end{aligned}
$$


in which the input $u$ is used to estimate $\eta_{1}$. As we show below, $u$ is designed following ideas reminiscent of the equivalent control-input notion that is, $u=\eta_{1}$ on the sliding mode $-c f$. [24, 11]. Furthermore, we use certaintyequivalence principle and the terms $H_{1} f(\hat{x})$ and $\frac{1}{2} \hat{\beta} H_{1} M(y-C \hat{x})$ to compensate for the effect of the Lipschitz non-linearities. However, since the Lipschitz constant $K_{f}$ is unknown, we use the adaptation law

$$
\dot{\hat{\beta}}=\gamma_{1}|M(y-C \hat{x})|^{2}-\sigma_{2} \hat{\beta}
$$

where $\gamma_{1}$ and $\sigma_{2}>0, \hat{\beta}$ is the estimate of a parameter $\beta$ which is defined below and dominates over the effect of the Lipschitz nonlinearity and we define $M$ as follows. Let $G=N C \in \mathbb{R}^{q_{1} \times\left(n+q_{2}\right)}$ with $N \in \mathbb{R}^{q_{1} \times p}$ be s.t. $G H_{2}$ is nonsingular and

$$
\begin{aligned}
A_{G} & =\left[I-H_{2}\left(G H_{2}\right)^{-1} G\right] P A \\
B_{G} & =\left[I-H_{2}\left(G H_{2}\right)^{-1} G\right] H_{1} .
\end{aligned}
$$

After [9], if (and only if) for each complex number $\lambda$ such that $\operatorname{Re}(\lambda) \geq 0$,

$$
\begin{aligned}
& \operatorname{rank}\left(C B_{G}\right)=\operatorname{rank}\left(B_{G}\right) \\
& \operatorname{rank}\left(\begin{array}{cc}
A_{G}-\lambda I & B_{G} \\
C & 0
\end{array}\right)=n+\operatorname{rank}\left(B_{G}\right)
\end{aligned}
$$

then, for any positive definite symmetric matrix $Q$ there exist $P_{G}=P_{G}^{\top}, L$ and $M$ such that

$$
\begin{gathered}
{\left[A_{G}-L C\right]^{\top} P_{G}+P_{G}\left[A_{G}-L C\right]=-Q} \\
P_{G} B_{G}=(M C)^{\top} .
\end{gathered}
$$

To solve (22), (23), we find the smallest $\rho_{*}$ such that

$$
\begin{gathered}
P_{G}>0 \\
P_{G} A_{G}+A_{G}^{\top} P_{G}+R C+C^{\top} R^{\top}<0 \\
{\left[\begin{array}{cc}
\rho_{*} I & B_{G}^{\top} P_{G}-M C \\
P_{G} B_{G}-C^{\top} M^{\top} & \rho_{*} I
\end{array}\right] \geq 0 .}
\end{gathered}
$$

The solution to the latter yields the minimum $\rho_{*}=0$ such that $P_{G}, M, R$ and $L=-P_{G}^{-1} R$ satisfy (22) and (23).

We carry on the estimator design by introducing a discontinuous input $u$ with the aim at steering the error trajectories into an invariant manifold in which $e(t)$ converges to zero. Define the sliding manifold $\{S \equiv 0\}$ where

$$
S(t):=N C e(t)+\int_{0}^{t} G L C e(\tau) d \tau
$$

which is equivalent to

$$
\dot{S}=G \dot{e}+G L C e .
$$

We premultiply (4a) by $P$ and subtract (15). Then, proceeding as in Section 2.1, we obtain

$$
\begin{array}{rl}
\dot{e}=P & A e-L C e-\frac{1}{2} \hat{\beta} H_{1} M(y-C \hat{x})+H_{1}[f(x)-f(\hat{x})] \\
+ & H_{2}\left(\eta_{1}-u\right)
\end{array}
$$

where we used (13) -we recall that $v_{1}$ is a virtual input used only in the analysis. Replacing (29) in (28), we obtain

$$
\begin{gathered}
\dot{S}=G(P A-L C) e+G H_{1}[f(x)-f(\hat{x})]+G H_{2}\left(\eta_{1}-u\right) \\
-\frac{1}{2} \hat{\beta} G H_{1} M(y-C \hat{x})+G L C e
\end{gathered}
$$


Next, let

$$
\begin{array}{r}
u=\left(G H_{2}\right)^{-1}\left[(\delta+\hat{\rho}) \frac{S}{|S|}-G P A \hat{x}-G H_{1} f(\hat{x})\right. \\
\left.-\frac{1}{2} G H_{1} \hat{\beta} M(y-C \hat{x})\right]
\end{array}
$$

where $\hat{\rho}$ is a design gain, updated according to

$$
\dot{\hat{\rho}}=\gamma_{2}|S|-\sigma \hat{\rho}
$$

Then, if we use (31) in (30) we obtain

$$
\begin{aligned}
\dot{S} & =-(\hat{\rho}+\delta) \frac{S}{|S|}+\Phi\left(\eta_{1}, x\right) \\
\Phi\left(\eta_{1}, x\right) & :=G H_{2} \eta_{1}+G P A x+G H_{1} f(x) .
\end{aligned}
$$

Now, in view of Assumption 1.1, $\varphi(t):=\Phi\left(\eta_{1}(t), x(t)\right)$ is bounded, let this bound be

$$
\rho:=\sup _{t \geq 0}|\varphi(t)|
$$

Define $\tilde{\rho}=\rho-\hat{\rho}$ and consider now the positive definite radially unbounded function

$$
V_{1}(S, \tilde{\rho})=\frac{1}{2}|S|^{2}+\frac{1}{2 \gamma_{2}} \tilde{\rho}^{2} ;
$$

its total time derivative along the trajectories of (33a) and (32) yields

$$
\begin{aligned}
\dot{V}_{1} & \leq|\varphi(t)||S|-(\hat{\rho}+\delta)|S|+\tilde{\rho}\left[-|S|+\frac{\sigma}{\gamma_{2}}(\rho-\tilde{\rho})\right] \\
& \leq-\delta|S|-\frac{\sigma}{\gamma_{2}} \tilde{\rho}^{2}+\frac{\sigma}{\gamma_{2}} \rho \tilde{\rho}
\end{aligned}
$$

which implies that the trajectories are bounded. To see this, note that using the triangle inequality $2 \rho \tilde{\rho} \leq \rho^{2}+\tilde{\rho}^{2}$, we have $\dot{V} \leq-\delta|S|-\frac{\sigma}{2 \gamma_{2}} \tilde{\rho}^{2}+\frac{\sigma}{2 \gamma_{2}} \rho^{2}$, that is, $\dot{V} \leq 0$ for large values of $\left[|S|,|\tilde{\rho}|^{2}\right]^{\top}$-see [17].

Corollary 2.1 Let $\Omega$ be a compact set and assume that $x(t) \in \Omega$ for all $t$. Consider the estimator given by (16), (31) and (17) and let $\hat{\rho} \equiv \rho$. Then, the sliding mode $\{S=0\}$ is reached in finite time.

The proof of the first claim follows directly from the previous computations, observing that $V_{1}(S)$ is a quadratic function of $S$ and its derivative is negative definite and satisfies $\dot{V}_{1} \leq-2 \delta V_{1}^{1 / 2}$.

Remark 2.1 Although the statement of the corollary does not establish necessity it is also implicit in the previous computations that only in the case that the parameter $\rho$ is known, the sliding manifold may be reached (in finite time).

\subsection{Convergence of estimation errors}

Now we investigate the dynamic behavior of the error trajectories on the sliding manifold. Solving $(30)$ for $\left(\eta_{1}-u\right)$, we obtain

$$
\begin{aligned}
\left(\eta_{1}-u\right)=\left(G H_{2}\right)^{-1}[ & \frac{1}{2} \hat{\beta} G H_{1} M C e-G L C e \\
& \left.\quad-G H_{1}[f(x)-f(\hat{x})]-G K e+\dot{S}\right]
\end{aligned}
$$

where we also used $K=P A-L C$. We replace $\left(\eta_{1}-u\right)$ by the right-hand side of (37) in (29) to obtain

$$
\dot{e}=\left[A_{G}-L C\right] e+B_{G}\left[f(x)-f(\hat{x})-\frac{1}{2} \hat{\beta} M C e\right]+H_{2}\left(G H_{2}\right)^{-1} \dot{S}
$$


Let $\beta$ be such that

$$
\operatorname{det}\left|\begin{array}{cc}
\frac{\lambda_{m}(Q)}{2} & K_{f} \\
K_{f} & \beta
\end{array}\right| \geq 0
$$

and define $\tilde{\beta}=\beta-\hat{\beta}$ then, the total derivative of the positive definite radially unbounded continuous function

$$
V_{2}(e, \tilde{\beta})=e^{\top} P_{G} e+\frac{1}{2 \gamma_{1}} \tilde{\beta}^{2}
$$

along the estimation error trajectories (38) and

$$
\dot{\tilde{\beta}}=-\gamma_{1}|M(y-C \hat{x})|^{2}+\sigma_{2} \hat{\beta}
$$

yields

$$
\begin{array}{r}
\dot{V}_{2} \leq-e^{\top} Q e+2 e^{\top} P_{G} B_{G}\left[f(x)-f(\hat{x})-\frac{1}{2} \beta M C e\right] \\
-2 e^{\top} P_{G} H_{2}\left(G H_{2}\right)^{-1} \dot{S}+\frac{1}{\gamma_{1}} \tilde{\beta}\left[\sigma_{2}(\beta-\tilde{\beta})\right],
\end{array}
$$

for which we used (22) and (23). Furthermore, using once more (23) as well as (2), we obtain

$$
\begin{aligned}
\dot{V}_{2} \leq-\lambda_{m}(Q) & |e|^{2}-\beta|M C e|^{2}+2 K_{f}|e||M C e| \\
& +2\left|P_{G} H_{2}\left(G H_{2}\right)^{-1}\right||e||\dot{S}|-\frac{\sigma_{2}}{\gamma_{1}} \tilde{\beta}^{2}+\frac{\sigma_{2}}{\gamma_{1}} \beta \tilde{\beta}
\end{aligned}
$$

hence,

$$
\dot{V}_{2} \leq-\frac{\lambda_{m}(Q)}{2}|e|^{2}-\left(\begin{array}{c}
|e| \\
|M C e|
\end{array}\right)^{\top}\left(\begin{array}{cc}
\frac{\lambda_{m}(Q)}{2} & -K_{f} \\
-K_{f} & \beta
\end{array}\right)\left(\begin{array}{c}
|e| \\
|M C e|
\end{array}\right)+2\left|P_{G} H_{2}\left(G H_{2}\right)^{-1}\right||e||\dot{S}|-\frac{\sigma_{2}}{\gamma_{1}} \tilde{\beta}^{2}+\frac{\sigma_{2}}{\gamma_{1}} \beta \tilde{\beta}
$$

Next, we use the definition of the unknown constant $\beta$ given above by Equation (39) to obtain

$$
\dot{V}_{2} \leq-\frac{\lambda_{m}(Q)}{2}|e|^{2}+2\left|P_{G} H_{2}\left(G H_{2}\right)^{-1}\right||e||\dot{S}|-\frac{\sigma_{2}}{\gamma_{1}} \tilde{\beta}^{2}+\frac{\sigma_{2}}{\gamma_{1}} \beta \tilde{\beta}
$$

so in view of (39) and introducing $c_{b}$ such that $\left|P_{G} H_{2}\left(G H_{2}\right)^{-1}\right| \leq c_{b}$ hence $2 c_{b}|e||\dot{S}| \leq c_{b}^{2}|\dot{S}|^{2}+|e|^{2}$, we obtain

$$
\dot{V}_{2} \leq-\left[\frac{\lambda_{m}(Q)}{2}-1\right]|e|^{2}+c_{b}^{2}|\dot{S}|^{2}-\frac{\sigma_{2}}{2 \gamma_{1}} \tilde{\beta}^{2}+\frac{\sigma_{2}}{2 \gamma_{1}} \beta^{2} .
$$

We conclude that on the sliding manifold $\{S \equiv 0\}$, the trajectories $e(t)$ converge to a small neighborhood of the origin with radius dependent on $\beta, \sigma_{2}$ and $\gamma_{1}$, provided that $\lambda_{m}(Q)>2$-see [17].

In the particular case that the gain $\sigma_{2}=0$ or if $\beta$ is known (44) becomes

$$
\dot{V}_{2} \leq-\left[\frac{\lambda_{m}(Q)}{2}-1\right]|e|^{2}
$$

hence, one may conclude by invoking Barbălat's lemma, that $e(t)$ converges to zero asymptotically provided that the trajectories are on the sliding manifold. Although this is unfeasible, this observation helps us to establish our main theorem. 


\section{Main result}

In previous sections we showed that in the case that the parameters $\rho$ and $\beta$ are known, the sliding manifold is reached in finite time and the estimation errors converge asymptotically to zero ${ }^{4}$. In the scenario considered in this paper this cannot be guaranteed. Nonetheless, the error trajectories converge and remain arbitrarily close to the sliding manifold and consequently, they tend to an arbitrarily small compact set which contains the origin. We establish this next.

Theorem 1 For the system (1) under Assumption 1.1 consider the estimator defined by (17), (27), (32) and the continuously-implemented counter-part of the input u in (31),

$$
u=\left(G H_{2}\right)^{-1}\left[\delta S+\hat{\rho} \frac{S}{\varepsilon+|S|}-G P A \hat{x}-G H_{1} f(\hat{x})-\frac{1}{2} G H_{1} \hat{\beta} M(y-C \hat{x})\right], \quad \varepsilon>0 .
$$

Let (20), (21) hold and

$$
\begin{aligned}
& I+E C-P T=0 \\
& \quad H_{1}=P B \\
& \quad H_{2}=P F \\
& K=P A-L C \quad \text { is Hurwitz } \\
& J=L-K E .
\end{aligned}
$$

Then, the estimation error trajectories converge to a compact set centered at $e=0$ and which may be diminished at will for large values of $\gamma_{1}$ and $\delta$.

Remark 3.1 The conditions of the theorem clearly restrict the class of systems for which the proposed unknown-input observer applies. To satisfy all the conditions the estimator parameters are computed in the following order:

(1) $P$ and $E$ are generated by Eq. (8) with $X=[P E], R_{1}=\left[T^{\top}-C^{\top}\right]^{\top}$, and $R_{2}=I_{n+q_{2}}$;

(2) $H_{1}$ and $H_{2}$ are defined by Eqs. (46b) and (46c);

(3) $\mathrm{N}$ and $\mathrm{G}$ are selected such that $\mathrm{GH}_{2}=\mathrm{NCH}_{2}$ is nonsingular;

(4) $A_{G}$ and $B_{G}$ are generated via (18) and (19) subject to the structural conditions (20), (21);

(5) $L=-P_{G}^{-1} R$ where $P_{G}$ and $M$ are generated by the solution of the optimization problem described above by Equations (24)-(26);

(6) $K$ is defined by (46d);

(7) $J$ is defined by (46e).

\section{Proof of Theorem 1.}

The proof builds upon the material of the previous section. Roughly, we first show along the lines of Section 2.2, that $S(t)$ and $\tilde{\rho}(t)$ tend to an arbitrarily small ball. Secondly, we show the same property for $\dot{S}(t)$ so that asymptotic practical stability may be concluded, in view of (44). Furthermore, instrumental in the proof (to show convergence of $\dot{S}$ ) is the following technical lemma that follows as a corollary from [16, Theorem 2].

Lemma 2 Consider the ordinary differential equation

$$
\dot{\zeta}(t)=-\delta \zeta(t)+\nu(t), \quad t_{0} \in \mathbb{R}_{+}, \zeta\left(t_{0}\right)=\zeta_{o} \in \mathbb{R}^{n}, \delta>0
$$

where $\nu$ is uniformly continuous and bounded on its domain, $\mathbb{R}_{+}$. Then,

$$
\lim _{\delta \rightarrow+\infty} \dot{\zeta}(t, \delta)=0
$$

uniformly for all $t>t_{0}$.

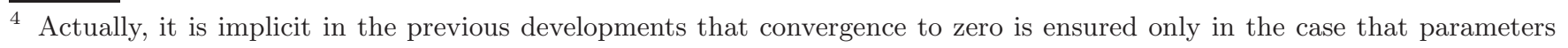
are known.
} 
1.- Convergence of $S(t)$ and $\tilde{\rho}(t)$.

After the development in Section 2.3, the estimation error trajectories satisfy (38) hence, (44). On the other hand, the dynamics of the sliding surface is defined by (30). Replacing in the latter the expression of $u$ given in (45), yields

$$
\dot{S}=-\delta S+\Phi\left(\eta_{1}, x\right)-\hat{\rho} \frac{S}{\varepsilon+|S|} .
$$

The total time derivative of $V_{1}$ defined in (35), using (49) and $\hat{\rho}=\rho-\tilde{\rho}$, satisfies

$$
\begin{aligned}
\dot{V}_{1} \leq S^{\top} & {[-\delta S+\varphi(t)]+\frac{\tilde{\rho}}{\gamma_{2}}\left[-\gamma_{2}|S|+\sigma(\rho-\tilde{\rho})\right] } \\
& +(\rho-\tilde{\rho})\left[|S|-\frac{|S|^{2}}{|S|+\varepsilon}\right] \\
\leq- & \delta|S|^{2}-\frac{\sigma}{\gamma_{2}} \tilde{\rho}^{2}+\frac{\sigma}{\gamma_{2}} \rho \tilde{\rho}+(\rho-\tilde{\rho}) \frac{\varepsilon|S|}{|S|+\varepsilon}
\end{aligned}
$$

and in view of the inequalities

$$
\begin{gathered}
\frac{|S|}{|S|+\varepsilon} \leq 1 \\
\rho \tilde{\rho} \leq \frac{1}{2}\left(\rho^{2}+\tilde{\rho}^{2}\right) \\
\tilde{\rho} \varepsilon=\left(\tilde{\rho}\left[\frac{\sigma}{2 \gamma_{2}}\right]^{1 / 2}\right)\left(\varepsilon\left[\frac{2 \gamma_{2}}{\sigma}\right]^{1 / 2}\right) \leq \frac{1}{2}\left[\frac{\tilde{\rho}^{2} \sigma}{2 \gamma_{2}}+\frac{2 \varepsilon^{2} \gamma_{2}}{\sigma}\right]
\end{gathered}
$$

we see that

$$
\dot{V}_{1} \leq-\delta|S|^{2}-\frac{\sigma}{4 \gamma_{2}} \tilde{\rho}^{2}+\frac{\sigma}{2 \gamma_{2}} \rho^{2}+\rho \varepsilon+\varepsilon^{2} \frac{\gamma_{2}}{\sigma},
$$

where the last three terms on the right-hand side are constant. Next, define

$$
c_{2}\left(\rho, \varepsilon, \gamma_{2}, \sigma\right):=\frac{\sigma}{2 \gamma_{2}} \rho^{2}+\rho \varepsilon+\varepsilon^{2} \frac{\gamma_{2}}{\sigma},
$$

then

$$
\dot{V}_{1} \leq-\min \left\{2 \delta, \frac{\sigma}{2}\right\}\left[\frac{|S|^{2}}{2}+\frac{\tilde{\rho}^{2}}{2 \gamma_{2}}\right]+c_{2} .
$$

That is, defining $K_{0}:=\min \left\{2 \delta, \frac{\sigma}{2}\right\}$ we see that the trajectories satisfy

$$
\dot{V}_{1}(S(t), \tilde{\rho}(t)) \leq-K_{0} V_{1}(S(t), \tilde{\rho}(t))+c_{2}
$$

so by integrating on both sides of the latter and invoking the comparison theorem, it follows that

$$
\lim _{t \rightarrow \infty} V_{1}(S(t), \tilde{\rho}(t)) \leq \frac{c_{2}\left(\rho, \varepsilon, \gamma_{2}, \sigma\right)}{K_{0}(\sigma, \delta)}
$$

Let $\varepsilon \propto\left(1 / \gamma_{2}\right)$, then the quotient $c_{2} / K_{0}$ can be arbitrarily diminished by enlarging $\gamma_{2}$ and $\delta$. It is concluded that $S(t)$ and $\tilde{\rho}(t)$ tend asymptotically to an arbitrarily small compact set centered at $\{S=0, \tilde{\rho}=0\}$.

2.- Convergence of $\dot{S}(t)$

We invoke Lemma 2 with $\zeta=S$ and $\nu(t)=\tilde{\nu}(t, \hat{\rho}(t), S(t))$ where

$$
\tilde{\nu}(t, \hat{\rho}, S):=\varphi(t)-\hat{\rho} \frac{S}{|S|+\varepsilon} .
$$

To that end, we first show that $\nu(t)$ is bounded and uniformly continuous in $t$. 
Uniform boundedness of $\nu(t)$ follows from boundedness of $\tilde{\rho}(t)$ hence of $\hat{\rho}(t)=\rho-\tilde{\rho}(t)$, of $S(t)$ and of $\varphi(t)$-see $(34)$. Uniform continuity follows from the fact that $\dot{\nu}(t)$ is also bounded. To see the latter we write

$$
\dot{\tilde{\nu}}=\dot{\varphi}(t)-\dot{\hat{\rho}} \frac{S}{|S|+\varepsilon}-\hat{\rho} F(S) \dot{S}
$$

where

hence

$$
F(S)=\frac{\varepsilon}{(|S|+\varepsilon)^{2}} \leq \frac{1}{\varepsilon}
$$

$$
\dot{\nu}(t) \leq|\dot{\varphi}(t)|+|\dot{\tilde{\rho}}(t)|+\frac{1}{\varepsilon}[\rho+|\tilde{\rho}(t)|]|\dot{S}(t)| .
$$

To investigate the boundedness of $\dot{\nu}(t)$ we analyze the right-hand side of (54) term by term. After (49)

$$
\dot{S}(t)=-\delta S(t)+\varphi(t)-[\rho-\tilde{\rho}(t)] \frac{S(t)}{|S(t)|+\varepsilon}
$$

and since $S(t), \tilde{\rho}(t)$ and $\varphi(t)$ are bounded so is $\dot{S}(t)$. In addition, the norm of

$$
\dot{\varphi}(t)=G H_{2} \dot{\eta}_{1}(t)+G P A \dot{x}(t)+G H_{1} \frac{\partial f}{\partial x}(x(t)) \dot{x}(t)
$$

is bounded in view of Assumption 1.1. Finally, we observe from (32) that

$$
|\dot{\tilde{\rho}}(t)| \leq \sigma \rho+\gamma_{2}|S(t)|+\sigma|\tilde{\rho}(t)|
$$

which is bounded for all $t \geq 0$ as well. We conclude that $\dot{\nu}(t)$ is bounded and consequently, $\nu(t)$ is uniformly continuous.

Next, from Lemma 2, it follows that

$$
\lim _{\delta \rightarrow \infty} \dot{S}(t, \delta)=0
$$

uniformly for all $t>t_{0} \geq 0$. That is, for any $\Delta_{c}>0$, there exists $\delta_{c}>0$ such that for all $\delta \geq \delta_{c}$,

$$
|\dot{S}(t)| \leq \Delta_{c} \quad \forall t>t_{0}
$$

\section{3.- Convergence of $e(t)$}

We recall the function $V_{2}$ defined in (40). Its total derivative satisfies (44) regardless of the definition of $u$ and whether the sliding mode is achieved or not. Let $p_{m}>0$ be such that $e^{\top} P_{G} e \geq p_{m}|e|^{2}$. Let $\Delta_{c}>0$ : then, there exists $\delta_{c}$ such that Inequality (55) is satisfied, and consequently we have

$$
\begin{aligned}
\dot{V}_{2} \leq-\sigma_{2} & \left(\frac{1}{2 \gamma_{1}} \tilde{\beta}^{2}\right)-\frac{\lambda_{m}(Q)-2}{p_{m}}\left(\frac{1}{2} p_{m}|e|^{2}\right) \\
& +\underbrace{\frac{\sigma_{2}}{2 \gamma_{1}} \beta^{2}+\Delta_{c}^{2} c_{b}^{2}}_{c_{3}} \\
& \leq-\min \left\{\sigma_{2}, \frac{\lambda_{m}(Q)-2}{p_{m}}\right\} V_{2}+c_{3}\left(\delta, \gamma_{1}, \sigma_{2}\right) .
\end{aligned}
$$

Integrating on both sides of the latter along the trajectories, from $t_{0}$ to $t$ we obtain that $V_{2}(e(t), \tilde{\beta}(t))$ tends asymptotically to the compact set

$$
\left\{(e, \tilde{\beta}) \in \mathbb{R}^{n+q_{2}} \times \mathbb{R}: V_{2}(e, \tilde{\beta}) \leq \frac{c_{3}}{\min \left\{\sigma_{2}, \frac{\lambda_{m}(Q)-2}{p_{m}}\right\}}\right\} .
$$


Note that the previous upper-bound on $V_{2}(e, \tilde{\beta})$ may be reduced at will for any fixed $\sigma_{2}$, by enlarging $\gamma_{1}$ and $\delta$.

The statement of the theorem follows.

\subsection{Reconstruction of the unknown input $\eta_{1}$}

That the unknown input $\eta_{1}$ may be reconstructed with arbitrary accuracy follows as a direct corollary from the results established previously.

Corollary 3 For any tolerance $\varepsilon_{\eta}>0$ there exist gains $\delta, \gamma_{1}, \gamma_{2}, \sigma_{2}$ and $0<T_{\eta}<\infty$ such that

$$
\left|u(t)-\eta_{1}(t)\right| \leq \varepsilon_{\eta} \quad \forall t \geq t_{0}+T_{\eta}
$$

Proof. From (37) we have

$$
\left|u(t)-\eta_{1}(t)\right| \leq\left|\left(G H_{2}\right)^{-1}\right|\left[\frac{1}{2}|\hat{\beta}(t)|\left|G H_{1}\right||M C e(t)|+|G L C e(t)|+\left|G H_{1} K_{f}\right||e(t)|+|G K||e(t)|+|\dot{S}(t)|\right] .
$$

We have showed that $\hat{\beta}(t)=\beta-\tilde{\beta}(t)$ is bounded and all terms on the right hand side of the expression above are factors of $e(t)$ and $\dot{S}(t)$ which converge to an arbitrarily small compact set centered at the origin. The statement of the corollary follows.

\section{Numerical examples}

For the sake of illustration we consider two numerical examples which are illustrative of different scenarios where our theoretical results are useful. The first consists in the state estimation of a robot arm with noisy measurements and under the influence of unknown friction. The second example consists in a problem of synchronization of chaotic systems with application to the secured transmission of information.

\subsection{Single-link flexible-joint robot arm}

We consider the model of a single-link robot arm with flexible joint, excited by a sinusoidal input i.e.,

$$
\begin{aligned}
J_{2} \ddot{\theta}_{2}+F_{2} \dot{\theta}_{1}+K\left(\theta_{2}-\theta_{1}\right)+m g l \cos \left(\theta_{2}\right) & =0 \\
J_{1} \ddot{\theta}_{1}+F_{1} \dot{\theta}_{1}+K\left(\theta_{1}-\theta_{2}\right) & =K_{t} \sin (t)
\end{aligned}
$$

where $\theta_{1}$ and $\theta_{2}$ are the angular rotations of the motor and the link, respectively. The parameters of the robot arm are as follows: $J_{1}=3.7 \times 10^{-3} \mathrm{kgm}^{2}$ and $J_{2}=3.7 \times 10^{-3} \mathrm{kgm}^{2}$ are the motor and the link inertias, respectively; $m=2.1 \times 10^{-1} \mathrm{~kg}$ is the pointer mass, $l=0.15 \mathrm{~m}$ is the position of the center of gravity of the link, $K=0.18 \mathrm{Nms} / \mathrm{rad}$ is the joint stiffness constant, $F_{1}=4.6 \times 10^{-3} \mathrm{Nms} / \mathrm{rad}$ and $F_{2}=6.4 \times 10^{-3} \mathrm{Nms} / \mathrm{rad}$ represent respectively the viscous friction coefficients of the motor and the link, respectively and $K_{t}=32 \times 10^{-3} \mathrm{Nms} / \mathrm{rad}$ is an input gain.

We define the state $x_{*}=\left[x_{* 1}, x_{* 2}, x_{* 3}, x_{4 *}\right]^{\top}$ where $x_{1 *}=\theta_{1}, x_{2 *}=\theta_{2}, x_{3 *}=\dot{\theta}_{1}$ and $x_{4 *}=\dot{\theta}_{2}$. Let $y=\left[y_{1}=\right.$ $\left.x_{* 1}+\eta_{2}, y_{2}=x_{* 2}+x_{4 *}\right]^{\top}$ be the measured output vector where $\eta_{2}(t)$ represents the measurement noise (a zero-mean Gaussian noise) that is, as in [30], we assume that the angular rotation of the motor and the sum of the angular rotation and velocity of the link are measured. We recall that $x=\left[x_{*}, \eta_{2}\right]^{\top}$ is the augmented state. We assume that the viscous friction parameter $F_{2}$ is unknown which generates the unknown signal $\eta_{1}(t)=\frac{F_{2}}{J_{2}} \dot{\theta}_{2}$. Hence, the system (56) may be written in the form (1) with

$$
\begin{aligned}
& A_{0}=\left[\begin{array}{cccr}
0 & 0 & 1 & 0 \\
0 & 0 & 0 & 1 \\
-48.6486 & 48.6486 & -12.4324 & 0 \\
19.3548 & 619.3548 & 0 & 0
\end{array}\right], B=\left[\begin{array}{c}
0 \\
0 \\
0 \\
-33.1935
\end{array}\right], F=\left[\begin{array}{c}
0 \\
0 \\
0 \\
-1
\end{array}\right] \\
& C_{0}=\left[\begin{array}{llll}
1 & 0 & 0 & 0 \\
0 & 1 & 0 & 1
\end{array}\right], G_{0}=\left[\begin{array}{l}
1 \\
0
\end{array}\right], f_{0}\left(x_{*}\right)=\cos \left(x_{* 2}\right) \text {. }
\end{aligned}
$$


The design matrices are obtained following the procedure described in Remark 3.1:

$$
\begin{gathered}
E=\left[\begin{array}{cc}
0 & 0 \\
0 & -0.33 \\
0 & 0 \\
0 & -0.33 \\
-1 & 0
\end{array}\right], P=\left[\begin{array}{ccccc}
1.0000 & 0 & 0 & 0 \\
0 & 0.66 & 0 & -0.33 \\
0 & 0 & 1 & 0 \\
0 & -0.33 & 0 & 0.66 \\
-1 & 0 & 0 & 0
\end{array}\right], L=\left[\begin{array}{rr}
0.4955 & -0.0013 \\
0.0002 & 0.9969 \\
-24.3170 & 24.3251 \\
0.0001 & -0.0001 \\
0.4954 & 0.0011
\end{array}\right], \\
K=\left[\begin{array}{llllll}
-0.4955 & 0.0013 & 1 & 0.0013 & -0.4955 \\
-6.4512 & 5.4541 & 0 & -0.3302 & -0.0002 \\
-24.3316 & 24.3235 & -12.4324 & -24.3251 & 24.3170 \\
12.9037 & -12.9038 & 0 & -0.3332 & -0.0001 \\
-0.4954 & -0.0011 & -1 & -0.0011 & -0.4954
\end{array}\right], N=\left[\begin{array}{l}
0 \\
0
\end{array}\right] .
\end{gathered}
$$

The design parameters are chosen as follows: $\varepsilon=0.001, \delta=100, \sigma=100$ and $\gamma_{2}=1000$. The initial conditions are: $x(0)=[3,1,1,2,-0.8]^{\top}, \hat{x}(0)=[-2,0,2,4,2.17]^{\top}, \hat{\rho}=0$.

Simulation results are as follows. Figure 1 illustrates that the states and the measurement noise $\eta_{2}$ are efficiently estimated, hence the effect of noise is perfectly canceled (we recall that $e_{5}=x_{5}-\hat{x}_{5}$ represents the estimation error of the measurement noise). Figure 2 depicts that the unknown input $\eta_{1}$ is well reconstructed.

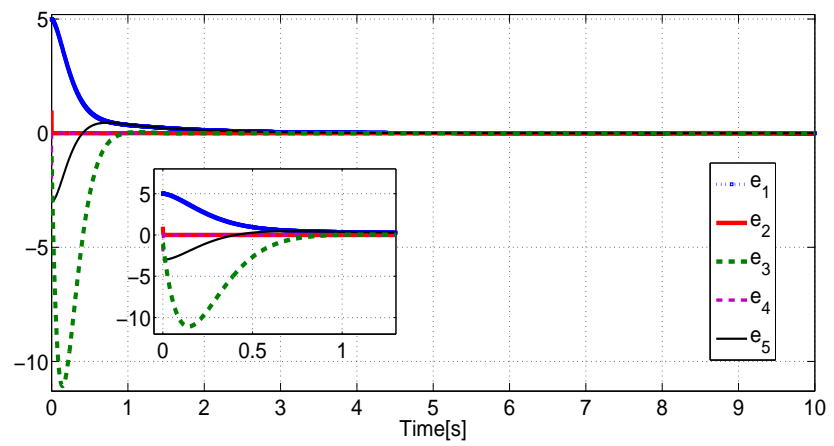

Fig. 1. Estimation errors $e_{1}=x_{1}-\hat{x}_{1}, e_{2}=x_{2}-\hat{x}_{2}, e_{3}=x_{3}-\hat{x}_{3}, e_{4}=x_{4}-\hat{x}_{4}$ and $e_{5}=x_{5}-\hat{x}_{5}$

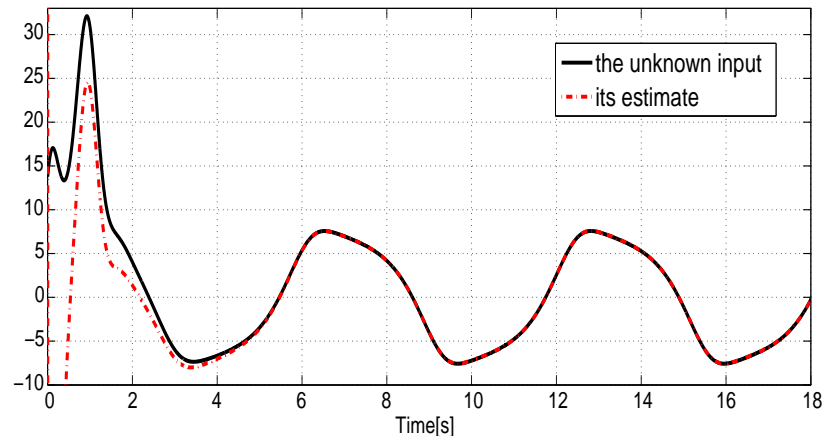

Fig. 2. The unknown input $\eta_{1}$ and its estimate

\subsection{Chaotic communication system with channel noise}

We present simulation results to test the performance of the proposed methodology. We take as case-study a problem of synchronization of chaotic systems, motivated by applications in the domain of secured communication $-c f$. [7]. 
The context is the following. A master system is designed to generate a chaotic carrier. Information is encoded and transmitted using such carrier. A receiver measures the transmitted signal and it is required to recover the encoded information. In the context of the unknown-input observers, the plant corresponds to the master system, the observer corresponds to the slave system. The unknown input corresponds to the encoded information to be recovered. It is assumed that the transmission channel is contaminated by noise, $\eta_{2}$.

The master system is a Lorenz chaotic oscillator subject to unknown inputs that is,

$$
\begin{aligned}
& \dot{x}_{* 1}=-a_{1} x_{* 1}+a_{2} x_{* 2} \\
& \dot{x}_{* 2}=r x_{* 1}-x_{* 2}-x_{* 1} x_{* 3}+\eta_{11} \\
& \dot{x}_{* 3}=x_{* 1} x_{* 2}-b x_{* 3}+\eta_{12}
\end{aligned}
$$

The measured outputs are

$$
\begin{array}{r}
y_{1}=x_{* 1}+\eta_{2} \\
y_{2}=2 x_{* 2}+\eta_{2} \\
y_{3}=x_{* 3}+\eta_{2} .
\end{array}
$$

The system (57) may be written in the form (1) with $x=\left[x_{* 1}, x_{* 2}, x_{* 3}\right]^{\top}$ as the state vector, $y=\left[y_{1}, y_{2}, y_{3}\right]^{\top}$ as the measured output vector, $\eta_{1}(t)=\left[\eta_{11}(t) ; \eta_{12}(t)\right]$ as the unknown inputs vector to be estimated, $\eta_{2}$ as noise and

$$
A_{0}=\left[\begin{array}{ccc}
-10 & 10 & 0 \\
28 & -1 & 0 \\
0 & 0 & -8 / 3
\end{array}\right], B=\left[\begin{array}{ll}
0 & 0 \\
1 & 0 \\
0 & 1
\end{array}\right], F=\left[\begin{array}{ll}
0 & 0 \\
1 & 0 \\
0 & 1
\end{array}\right], C_{0}=\left[\begin{array}{lll}
1 & 0 & 0 \\
0 & 2 & 0 \\
0 & 0 & 1
\end{array}\right], f_{0}\left(x_{*}\right)=\left[\begin{array}{c}
-x_{* 1} x_{* 3} \\
x_{* 1} x_{* 2}
\end{array}\right], \quad G_{0}=\left[\begin{array}{l}
1 \\
1 \\
1
\end{array}\right] .
$$

The unknown input is the transmitted message $\eta_{1}=\left[\eta_{11}, \eta_{12}\right]$ where $\eta_{11}$ and $\eta_{12}$ are assumed to be sinusoidal signals of respective amplitudes equal to $m_{1 \max }=1, m_{2 \max }=3$ and frequencies $f_{r 1}=0.33 \mathrm{~Hz}$ and $f_{r 2}=1.32 \mathrm{~Hz}$. The channel noise $\eta_{2}(t)$ consists on a zero mean Gaussian noise of frequency $10 \mathrm{~Hz}$ and variance equal to 1 , generated between lower and upper bounds respectively equal to -3 and 3 . We initialize the vector states of systems (57) at $x_{* 0}=[0.2,-0.4,-0.2]^{\top}$.

The system (57) presents chaotic behavior for $a_{1}=a_{2}=10, b=8 / 3$ and $r=28$ even in the presence of the inputs $\eta_{1}$, hence, the solutions are bounded and the Lipschitz extension transformation may be applied. Finally, the design constants are chosen as follows: $\varepsilon=0.0001, \delta=1000, \sigma=1000$ and $\gamma_{2}=10000$.

Note that in this example $B=F$ which leads to $B_{G}=0$ hence, $\hat{\beta}$ does not need to be updated. We initialize the states of the observer $(16)$ at $\hat{x}(0)=[-0.11,-0.14,0.2,-0.38]^{\top}$ and the adaptive parameter at $\hat{\rho}(0)=0$.

Simulation results illustrate that the effect of noise is perfectly canceled out (measurement noise is estimated) by the proposed receiver (observer) and the error synchronization is not affected-see Figure 3. Furthermore, despite the presence of noise in the public channel, the transmitted messages are well recovered by the receiver (16) as seen in Figure 4.

\section{Conclusion}

We presented robust unknown-input observer via continuously-implemented sliding modes. The estimator is able to reconstruct the unknown inputs and state with arbitrary accuracy. We have illustrated the utility and performance of the estimator via an example of a single-link robot and an application of a master-slave synchronization problem of chaotic systems for secured transmission of information.

\section{References}

[1] J. H. Ahrens and H. K. Khalil. High-gain observers in the presence of measurement noise: A switched-gain approach. Automatica, 45(4):936-943, 2009.

[2] V. Andrieu, L. Praly, and A. Astolfi. High gain observers with updated gain and homogeneous correction terms. Automatica, 45:422-428, 2009. 

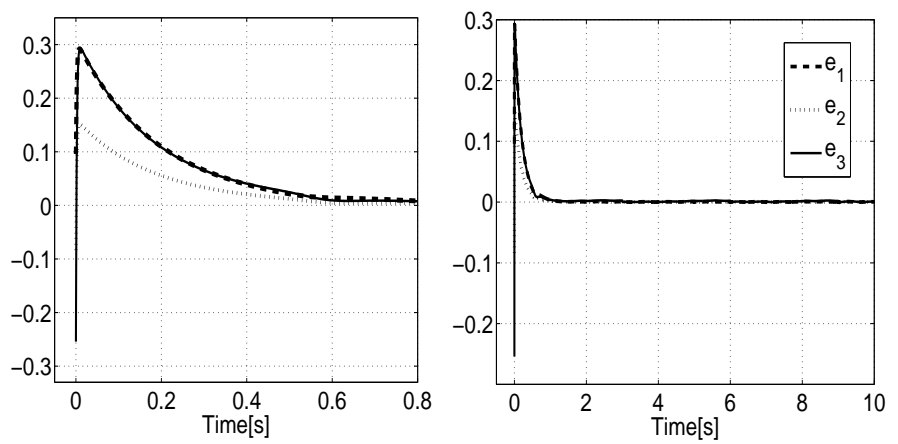

Fig. 3. Synchronization error $e_{1}=x_{1}-\hat{x}_{1}, e_{2}=x_{2}-\hat{x}_{2}$ and $e_{3}=x_{3}-\hat{x}_{3}$ in our methodology based on observer(16) in presence of noises and unknown inputs
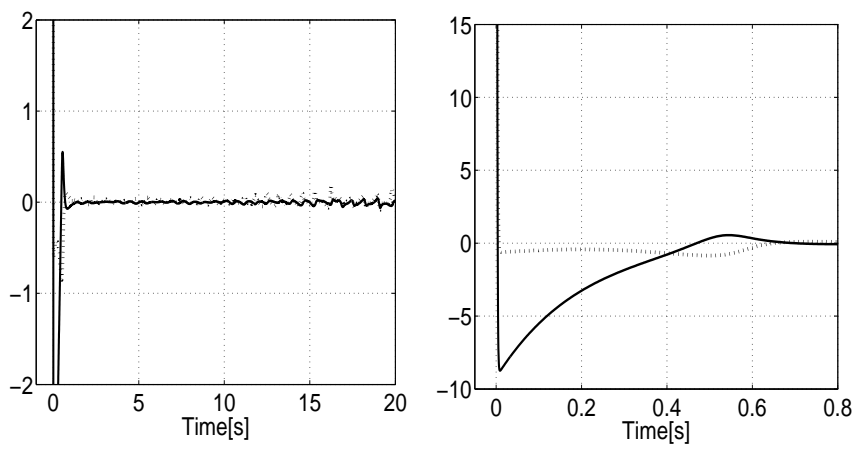

Fig. 4. Estimation errors for the unknown inputs $\eta_{11}$ and $\eta_{12}$ in presence of channel noise

[3] M. Arcak and P. Kokotović. Observer-based control of systems with slope-restricted nonlinearities. IEEE Trans. on Automat. Contr., 46(7):1146-1150, 2001.

[4] A.N. Atassi and H. Khalil. A separation principle for the stabilization of a class of nonlinear systems. IEEE Trans. on Automat. Contr., 44:1672-1687, 1999.

[5] J. P. Barbot, T. Boukhobza, and M. Djemai. Sliding mode observer for triangular input form. In Proc. of the 35th IEEE Conf. on Dec. and Contr., pages 936-943, Kobe, Japan, 1996.

[6] J. P Barbot and T. Floquet. Iterative higher-order sliding-mode observer design for nonlinear systems with unknown inputs. Technical report, INRIA, 2009. http://hal.inria.fr/inria-00442129.

[7] M. Boutayeb, M. Darouach, and H. Rafaralahy. Generalized state-space observers for chaotic synchronization and secure communication. IEEE Trans. on Circ. Syst. I: Fundamental Theory and Applications, 49(3):345-349, 2002.

[8] J. Chen and R. J. Patton. Robust Model-based Fault Diagnostic for Dynamic Systems. Kluwer, Mars 1999.

[9] M. Corless and J. Tu. State and input estimation for a class of uncertain systems. Automatica, 34:757-764, 1998.

[10] G. R. Duan, A. G. Wu, and W. N. Hou. Parametric approach fo luenberger observers for descriptor linear systems. Bull. of the Polish Academy of Sciences, 55(1):15-18, 2007.

[11] C. Edwards and S. K. Spurgeon. Sliding mode control: theory and applications. Taylor and Francis, London, 1998.

[12] C. Edwards, S. K. Spurgeon, and R.J. Patton. Sliding mode observers for fault detection and isolation. Automatica, 36:541-553, 2000.

[13] C. Edwards and C.P. Tan. A comparison of sliding mode and unknown input observers for fault reconstruction. In European Journal of Control, volume 16, pages 245-260, 2006.

[14] T. Floquet and J. P Barbot. A canonical form for the design of unknown input sliding mode observers, volume 334, "Advances in Variable Structure and Sliding mode control". Springer, Lecture Notes in Control and Information Science, Berlin, 2006. http://hal.inria.fr/inria-00293867.

[15] L. Fridman, J. Davila, and A. Levant. High-order sliding-mode observation for linear systems with unknown 
inputs. Nonlinear Analysis: Hybrid Systems, 5:189-205, 2011.

[16] K. Kalsi, J. Lian, S. Hui, and H. Zak. Sliding-mode observers for systems with unknown inputs: A high-gain approach. Automatica, 46:347-353, 2010.

[17] H. Khalil. Nonlinear systems. Prentice Hall, 3rd ed., New York, 2002.

[18] A. J. Krener and W. Respondek. Nonlinear observer with linearizable error dynamics. SIAM J. on Contr. and Opt., 30:197-216, 1985.

[19] H. Lei, J. F. Wei, and W. Lin. A global observer for observable autonomous systems with bounded solution trajectories. In 44th IEEE conference on decision and control and the European control conference, pages 1911 - 1916, 2005.

[20] A. Levant. Robust exact differentiation via sliding mode technique. Automatica, 34:379-384, 1998.

[21] A. Levant. Higher-order sliding modes, differentiation and output-feedback control. International Journal of Control, 76:924-941, 2003.

[22] D. Miller and J. Vale. Pole placement adaptive control with persistent jumps in the plant parameters. In Proc. of the 49th IEEE Conf. on Dec. and Contr., pages 2596-2601, Atlanta, GA, USA, 2010.

[23] R. J. Patton and S. Klinkhieo. An adaptive approach to active fault-tolerant control. The Open Automation and Control Systems Journal, 2:54-61, 2009.

[24] R. J Patton, D. Putra, and S. Klinkhieo. Friction compensation as a fault tolerant control problem. International Journal of Systems Science, 41(8):987-1001, 2010.

[25] A. M. Pertew, H. J. Marquez, and Q. Zhao. $\mathcal{H}_{\infty}$ synthesis of unknown input observers for non-linear lipschitz systems. International Journal of Control, 78(15):1155-1165, 2005.

[26] C. P. Tan and C. Edwards. Sliding mode observers for detection and reconstruction of sensor faults. Automatica, 38:1815-1821, 2002.

[27] B. L. Walcott and S. Zak. State observation of nonlinear uncertain dynamical systems. IEEE Transactions on Automatic Control, 32:166-170, 1987.

[28] G. Wheeler, C. Su, and Y. Stepanenko. Sliding mode controller with improved adaptation laws for the upper bounds on the norm of uncertainties. Automatica, 34:1657-1661, 1998.

[29] J.X. Xu, Q.W. Jia, , and T.H. Lee. On the design of a nonlinear adaptive variable structure derivative estimator. IEEE Transactions on Automatic Control, 45:1028-1033, 2000.

[30] X. Yan and C. Edwards. Nonlinear robust fault reconstruction and estimation using a sliding mode observer. Automatica, 43:1605-1614, 2007. 